\title{
Determinant Identities and the Geometry of Lines and Circles
}

\author{
Nicolae Anghel
}

\begin{abstract}
The focus of this note is the nontrivial determinant identities which typically underlie the complex analytic proofs of all the results in the plane geometry of lines and circles. After setting up a basic dictionary relating lines and circles to complex determinants we derive such identities in connection with four geometry problems: the Steiner line, a variant of Euler's nine-point circle, the Johnson-Tzitzeica circles, and an extension of a certain geometry problem, proposed at the 52nd International Mathematical Olympiad, Amsterdam 2011.
\end{abstract}

\section{Introduction}

When it comes to solving any geometry problem there is no substitute for the elegance and concision of a purely geometric argument. Yet, there are situations when the complexity of a problem dictates that an analytic approach is more desirable. Even regardless such complexity, almost always analytic methods can prove to be more revealing that initially intended. One only has to be open to introspection.

The purpose of this note is to reinforce such an analytic approach when dealing with geometric problems about lines and circles in a Euclidean plane. Certainly, there is a huge inventory of fascinating problems of this type in the

Key Words: Determinant, Line, Circle, Complex Numbers, Möbius Transformation, Steiner Line, Euler Nine-Point Circle, Johnson-Tzitzeica Circles, International Mathematical Olympiad.

2010 Mathematics Subject Classification: Primary 51M04, 51N20, 11C20; Secondary 15A24.

Received: March 2013

Revised: May 2013

Accepted: June 2013 
multi-millennial history of classical Euclidean geometry. Our starting point is the elementary observation that any geometric information involving lines and circles can be conveniently packaged in analytic information via determinant theory. Then, most of the time solving such a problem merely requires concluding that certain determinants vanish, given the vanishing of others. The novel observation here is that all these determinants are typically related by some nontrivial identity, which is valid in a more general set-up than the one required in the actual problem. So, while the solution to the problem appears naturally as a specialization of variables in an appropriate determinant identity, far more valuable seems to be the underlying identity.

It turns out that this plan works great if, adapted to the problem, a coordinate system in the plane is chosen so that key quantities can be circularly permuted. Also, it is better to work with complex numbers, rather than real ones. After a brief approach to lines and circles in a plane via determinant theory we will exemplify the method on four problems: the Steiner line, a variant of Euler's nine-point circle, the Johnson-Tzitzeica circles, and a certain geometry problem, presented at the 52nd International Mathematical Olympiad, Amsterdam 2011. Interestingly enough, this last problem was solved during the contest by only nine students [3], another reason to give more credit to analytic geometry in general.

It is worth pointing out that several analytic approaches could be considered, based of the type of representation of lines and circles preferred, such as those using homogeneous coordinates, barycentric or trilinear. It is only a matter of personal preference that we use 'the complex-number method'.

\section{Lines and Circles via Determinants}

Let $E$ be a Euclidean plane. After choosing a coordinate system, $E$ will be identified with $\mathbf{R}^{2}$, and then with $\mathbf{C}$, the complex number field. In applications, the passage from $E$ to $\mathbf{C}$ will be specific to the problem at hand.

If $a_{1}, a_{2}$ are two distinct complex numbers, elementary properties of determinants show that the line through $a_{1}$ and $a_{2}, l\left(a_{1}, a_{2}\right)$, consists in all the complex numbers $z$ such that

$$
\operatorname{det}\left[\begin{array}{ccc}
z & \bar{z} & 1 \\
a_{1} & \bar{a}_{1} & 1 \\
a_{2} & \bar{a}_{2} & 1
\end{array}\right]=0
$$

Consequently, three points $a_{1}, a_{2}, a_{3}$, are non-collinear if and only if (1) does not hold, when $z=a_{3}$.

Similarly, given three non-collinear points $a_{1}, a_{2}$, and $a_{3}$, the unique circle 
determined by them, $c\left(a_{1}, a_{2}, a_{3}\right)$, has equation

$$
\operatorname{det}\left[\begin{array}{cccc}
z \bar{z} & z & \bar{z} & 1 \\
a_{1} \bar{a}_{1} & a_{1} & \bar{a}_{1} & 1 \\
a_{2} \bar{a}_{2} & a_{2} & \bar{a}_{2} & 1 \\
a_{3} \bar{a}_{3} & a_{3} & \bar{a}_{3} & 1
\end{array}\right]=0 .
$$

Obviously, when $a_{1}, a_{2}, a_{3}$ are viewed as the vertices of a triangle, $c\left(a_{1}, a_{2}, a_{3}\right)$ is its associated circumcircle.

Properties of determinants show that the circle $c\left(a_{1}, a_{2}, a_{3}\right)$ has center

$$
\alpha=-\frac{\operatorname{det}\left[\begin{array}{lll}
a_{1} \bar{a}_{1} & a_{1} & 1 \\
a_{2} \bar{a}_{2} & a_{2} & 1 \\
a_{3} \bar{a}_{3} & a_{3} & 1
\end{array}\right]}{\operatorname{det}\left[\begin{array}{lll}
a_{1} & \bar{a}_{1} & 1 \\
a_{2} & \bar{a}_{2} & 1 \\
a_{3} & \bar{a}_{3} & 1
\end{array}\right]} \text { and radius } r=\frac{\left|\operatorname{det}\left[\begin{array}{lll}
a_{1}^{2} & a_{1} & 1 \\
a_{2}^{2} & a_{2} & 1 \\
a_{3}^{2} & a_{3} & 1
\end{array}\right]\right|}{\left|\operatorname{det}\left[\begin{array}{lll}
a_{1} & \bar{a}_{1} & 1 \\
a_{2} & \bar{a}_{2} & 1 \\
a_{3} & \bar{a}_{3} & 1
\end{array}\right]\right|} .
$$

More precisely, the two formulas (3) can be obtained by formally identifying the (first row) expansion of the determinant in (2) with the appropriately scaled version of the center-radius equation of a circle, given by

$$
\left((z-\alpha)(\bar{z}-\bar{\alpha})-r^{2}\right) \operatorname{det}\left[\begin{array}{lll}
a_{1} & \bar{a}_{1} & 1 \\
a_{2} & \bar{a}_{2} & 1 \\
a_{3} & \bar{a}_{3} & 1
\end{array}\right]=0 .
$$

Notice also that the numerator in the formula of $r$ is the absolute value of a Vandermonde determinant, and equals $\left|a_{1}-a_{2}\right|\left|a_{2}-a_{3}\right|\left|a_{3}-a_{1}\right|$.

The expression of $r$ itself is a manifestation of the principle we try to unveil in this note. Indeed, it can be obtained as a specialization of the following identity of determinants:

$$
\begin{aligned}
& \operatorname{det}\left[\begin{array}{lll}
z_{1}^{2} & z_{1} & 1 \\
z_{2}^{2} & z_{2} & 1 \\
z_{3}^{2} & z_{3} & 1
\end{array}\right] \operatorname{det}\left[\begin{array}{lll}
\zeta_{1}^{2} & \zeta_{1} & 1 \\
\zeta_{2}^{2} & \zeta_{2} & 1 \\
\zeta_{3}^{2} & \zeta_{3} & 1
\end{array}\right]+ \\
& \operatorname{det}\left[\begin{array}{lll}
z_{1} \zeta_{1} & z_{1} & \zeta_{1} \\
z_{2} \zeta_{2} & z_{2} & \zeta_{2} \\
z_{3} \zeta_{3} & z_{3} & \zeta_{3}
\end{array}\right] \operatorname{det}\left[\begin{array}{lll}
z_{1} & \zeta_{1} & 1 \\
z_{2} & \zeta_{2} & 1 \\
z_{3} & \zeta_{3} & 1
\end{array}\right]= \\
& \operatorname{det}\left[\begin{array}{lll}
z_{1} \zeta_{1} & z_{1} & 1 \\
z_{2} \zeta_{2} & z_{2} & 1 \\
z_{3} \zeta_{3} & z_{3} & 1
\end{array}\right] \operatorname{det}\left[\begin{array}{lll}
z_{1} \zeta_{1} & \zeta_{1} & 1 \\
z_{2} \zeta_{2} & \zeta_{2} & 1 \\
z_{3} y_{3} & \zeta_{3} & 1
\end{array}\right] .
\end{aligned}
$$

In (4), $z_{i}, \zeta_{i}, i=1,2,3$, have the meaning of independent complex variables, and then the expression for $r$ is easily obtained by setting $z_{i}=a_{i}$ and $\zeta_{i}=\bar{a}_{i}$ in it. 
DETERMINANT IDENTITIES AND

The main reason to work with $\mathbf{C}$ instead of $\mathbf{R}^{2}$ is the powerful interplay between lines and circles and the group of Möbius transformations on the Riemann sphere, $\mathbf{C}_{\infty}=\mathbf{C} \cup \infty$,

$$
\mathbf{C}_{\infty} \ni z \longmapsto \frac{a z+b}{c z+d} \in \mathbf{C}_{\infty}, \quad a, b, c, d \in \mathbf{C}, a d-b c \neq 0 .
$$

It is well-known [1] that lines and circles in $\mathbf{C}$, when conveniently identified with 'circles in $\mathbf{C}_{\infty}$ ', are preserved by the Möbius transformations.

This is just one of the many useful things relating geometry and Möbius transformations. Recall, for instance, that the unique Möbius transformation sending three distinct complex numbers $a_{1}, a_{2}$ and $a_{3}$ to 1,0 and $\infty$, respectively, denoted $z \longmapsto\left(z, a_{1}, a_{2}, a_{3}\right)$, the cross-ratio of $z, a_{1}, a_{2}$ and $a_{3}$, has the property that when $a_{1}, a_{2}$, and $a_{3}$ are collinear, then $z$ and $z^{*}$ are symmetrical with respect to $l\left(a_{1}, a_{2}, a_{3}\right)$ if and only if

$$
\left(z^{*}, a_{1}, a_{2}, a_{3}\right)=\overline{\left(z, a_{1}, a_{2}, a_{3}\right)} .
$$

\section{The Steiner Line}

Steiner. In the plane of a triangle $A_{1} A_{2} A_{3}$ consider an arbitrary point $M$. Let $M_{1}, M_{2}$, and $M_{3}$ be the points symmetrical to $M$, relative to the sides $A_{2} A_{3}, A_{3} A_{1}$, and $A_{1} A_{2}$, respectively. Then the points $M_{1}, M_{2}$, and $M_{3}$, are collinear if and only if $M$ belongs to the circumcircle of triangle $A_{1} A_{2} A_{3}$ (Figure 1).

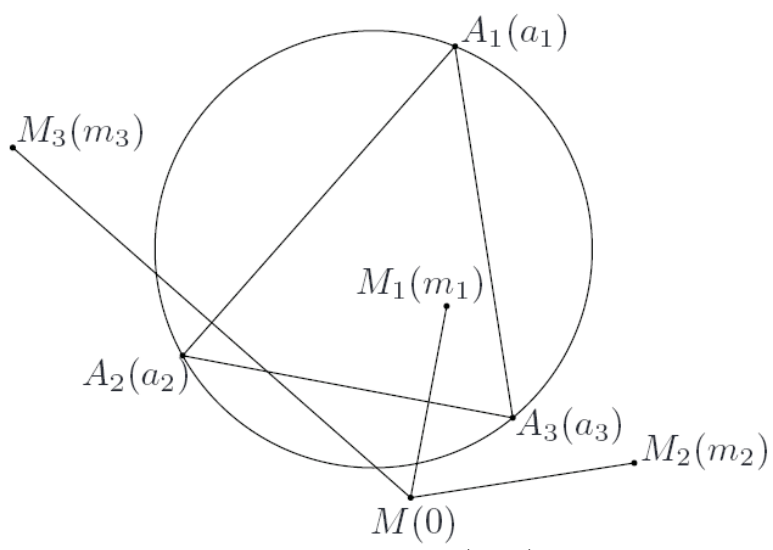

Figure 1: A Steiner (non)-line.

Choose a coordinate system with origin in $M$. Then $M$ has (complex) coordinate 0 , and $A_{1}, A_{2}$, and $A_{3}$, have coordinates, say, $a_{1}, a_{2}$, and $a_{3}$, 
DETERMINANT IDENTITIES AND

respectively. As coordinates of the vertices of a triangle they are mutually distinct. Also, if $m_{1}$ is the coordinate of $M_{1}$, equation (5) shows that

$$
\left(m_{1}, a_{2}, a_{3}, \infty\right)=\overline{\left(0, a_{2}, a_{3}, \infty\right)}, \text { which gives } m_{1}=\frac{a_{3} \bar{a}_{2}-a_{2} \bar{a}_{3}}{\bar{a}_{2}-\bar{a}_{3}},
$$

with similar expressions for $M_{2}\left(m_{2}\right)$ and $M_{3}\left(m_{3}\right)$. By (1) and (2) it suffices to show that

$$
\operatorname{det}\left[\begin{array}{lll}
m_{1} & \bar{m}_{1} & 1 \\
m_{2} & \bar{m}_{2} & 1 \\
m_{3} & \bar{m}_{3} & 1
\end{array}\right]=0 \text { if and only if } \operatorname{det}\left[\begin{array}{ccc}
a_{1} \bar{a}_{1} & a_{1} & \bar{a}_{1} \\
a_{2} \bar{a}_{2} & a_{2} & \bar{a}_{2} \\
a_{3} \bar{a}_{3} & a_{3} & \bar{a}_{3}
\end{array}\right]=0 .
$$

Now let us formally replace $a_{i}$ by $z_{i}$, and $\bar{a}_{i}$ by $\zeta_{i}, i=1,2,3$, in the second determinant appearing in (6). As before, for each $i, z_{i}$ and $\zeta_{i}$ should be interpreted as independent complex variables. In addition, assume the $z_{i}$ 's are pairwise distinct, and so are the $\zeta_{i}$ 's. These replacements suggest, at the level of $m_{i}$ 's, the following substitutions:

$$
m_{1} \longrightarrow \alpha_{1}:=\frac{z_{3} \zeta_{2}-z_{2} \zeta_{3}}{\zeta_{2}-\zeta_{3}}, \text { and } \bar{m}_{1} \longrightarrow \beta_{1}:=\frac{z_{2} \zeta_{3}-z_{3} \zeta_{2}}{z_{2}-z_{3}} \text {, etc. }
$$

The claim follows now from the following determinant identity:

$$
\begin{aligned}
& \operatorname{det}\left[\begin{array}{lll}
z_{1}^{2} & z_{1} & 1 \\
z_{2}^{2} & z_{2} & 1 \\
z_{3}^{2} & z_{3} & 1
\end{array}\right] \operatorname{det}\left[\begin{array}{lll}
\zeta_{1}^{2} & \zeta_{1} & 1 \\
\zeta_{2}^{2} & \zeta_{2} & 1 \\
\zeta_{3}^{2} & \zeta_{3} & 1
\end{array}\right] \operatorname{det}\left[\begin{array}{lll}
\alpha_{1} & \beta_{1} & 1 \\
\alpha_{2} & \beta_{2} & 1 \\
\alpha_{3} & \beta_{3} & 1
\end{array}\right]+ \\
& \operatorname{det}\left[\begin{array}{lll}
z_{1} \zeta_{1} & z_{1} & \zeta_{1} \\
z_{2} \zeta_{2} & z_{2} & \zeta_{2} \\
z_{3} \zeta_{3} & z_{3} & \zeta_{3}
\end{array}\right]\left(\operatorname{det}\left[\begin{array}{lll}
z_{1} & \zeta_{1} & 1 \\
z_{2} & \zeta_{2} & 1 \\
z_{3} & \zeta_{3} & 1
\end{array}\right]\right)=0 .
\end{aligned}
$$

\section{A Variant of Euler's Nine-Point Circle}

Euler. Let $H$ be the orthocenter of a triangle $A_{1} A_{2} A_{3}$. Let $P_{1}, P_{2}$, and $P_{3}$ be the points symmetrical to $H$, relative to the sides $A_{2} A_{3}, A_{3} A_{1}$, and $A_{1} A_{2}$, respectively. Similarly, let $Q_{1}, Q_{2}$, and $Q_{3}$ be symmetrical to $H$ relative to the mid-points of those sides. Then the nine points $A_{1}, A_{2}, A_{3}, P_{1}, P_{2}, P_{3}, Q_{1}$, $Q_{2}$, and $Q_{3}$, are concyclic (Figure 2).

Choose a coordinate system with origin in $H$, so $H$ has coordinate 0 . Assign then to $A_{1}, A_{2}$, and $A_{3}$, coordinates $a_{1}, a_{2}$, and $a_{3}$, respectively. Since $H$ is the orthocenter of the triangle $A_{1} A_{2} A_{3}$ we have $H A_{1} \perp A_{2} A_{3}, H A_{2} \perp A_{3} A_{1}$, and $H A_{3} \perp A_{1} A_{2}$, which analytically mean, respectively

$$
\operatorname{det}\left[\begin{array}{ccc}
a_{1} & \bar{a}_{1} & 0 \\
-a_{2} & \bar{a}_{2} & 1 \\
-a_{3} & \bar{a}_{3} & 1
\end{array}\right]=0, \operatorname{det}\left[\begin{array}{ccc}
a_{2} & \bar{a}_{2} & 0 \\
-a_{3} & \bar{a}_{3} & 1 \\
-a_{1} & \bar{a}_{1} & 1
\end{array}\right]=0, \operatorname{det}\left[\begin{array}{ccc}
a_{3} & \bar{a}_{3} & 0 \\
-a_{1} & \bar{a}_{1} & 1 \\
-a_{2} & \bar{a}_{2} & 1
\end{array}\right]=0 .
$$




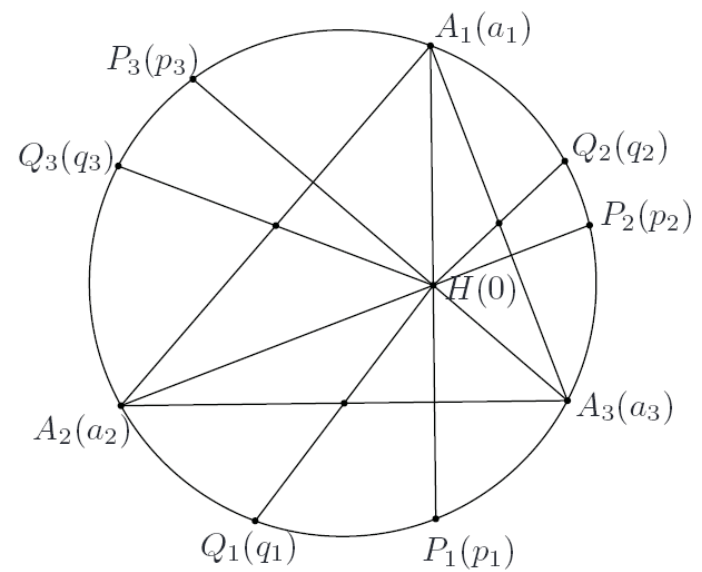

Figure 2: A variant of Euler's nine-point circle.

Evidently, the last two determinant equations in (7) could have been obtained from the first one by circular permutations.

Now the coordinate of $Q_{1}$ is clearly $q_{1}:=a_{2}+a_{3}$, and by circular permutations, we have similar expressions for the coordinates $q_{2}$ and $q_{3}$ of $Q_{2}$ and $Q_{3}$. The coordinate $p_{1}$ of $P_{1}$, is as before

$$
p_{1}=\frac{a_{3} \bar{a}_{2}-a_{2} \bar{a}_{3}}{\bar{a}_{2}-\bar{a}_{3}},
$$

with similar expressions for $P_{2}\left(p_{2}\right)$ and $P_{3}\left(p_{3}\right)$.

By (2), it suffices to show that in the presence of $(7), q_{1}, q_{2}$ and $q_{3}$, on one hand, and $p_{1}, p_{2}$ and $p_{3}$, on the other hand, belong to the circle $c\left(a_{1}, a_{2}, a_{3}\right)$. We will only show that $q_{1}$ and $p_{1}$ belong to that circle, with all the others following similarly.

Making the now familiar replacements $a_{i} \longrightarrow z_{i}$, and $\bar{a}_{i} \longrightarrow \zeta_{i}, i=1,2,3$, the determinants appearing in the three equations $(7)$, become $\operatorname{det}\left[\begin{array}{ccc}z_{1} & \zeta_{1} & 0 \\ -z_{2} & \zeta_{2} & 1 \\ -z_{3} & \zeta_{3} & 1\end{array}\right]:=\xi_{1}, \quad \operatorname{det}\left[\begin{array}{ccc}z_{2} & \zeta_{2} & 0 \\ -z_{3} & \zeta_{3} & 1 \\ -z_{1} & \zeta_{1} & 1\end{array}\right]:=\xi_{2}, \quad \operatorname{det}\left[\begin{array}{ccc}z_{3} & \zeta_{3} & 0 \\ -z_{1} & \zeta_{1} & 1 \\ -z_{2} & \zeta_{2} & 1\end{array}\right]:=\xi_{3}$

Our claim will follow from the following two identities, the first one applying to $q_{1}$, and the second to $p_{1}$ :

$$
\operatorname{det}\left[\begin{array}{cccc}
\left(z_{2}+z_{3}\right)\left(\zeta_{2}+\zeta_{3}\right) & z_{2}+z_{3} & \zeta_{2}+\zeta_{3} & 1 \\
z_{1} \zeta_{1} & z_{1} & \zeta_{1} & 1 \\
z_{2} \zeta_{2} & z_{2} & \zeta_{2} & 1 \\
z_{3} \zeta_{3} & z_{3} & \zeta_{3} & 1
\end{array}\right]=\frac{1}{2} \operatorname{det}\left[\begin{array}{ccc}
z_{1} & \zeta_{1} & \xi_{2}-\xi_{3} \\
z_{2} & \zeta_{2} & \xi_{2} \\
z_{3} & \zeta_{3} & -\xi_{3}
\end{array}\right],
$$


DETERMINANT IDENTITIES AND

$$
\begin{gathered}
\operatorname{det}\left[\begin{array}{cccc}
-\frac{\left(z_{3} \zeta_{2}-z_{2} \zeta_{3}\right)^{2}}{\left(z_{2}-z_{3}\right)\left(\zeta_{2}-\zeta_{3}\right)} & \frac{z_{3} \zeta_{2}-z_{2} \zeta_{3}}{\zeta_{2}-\zeta_{3}} & -\frac{z_{3} \zeta_{2}-z_{2} \zeta_{3}}{z_{2}-z_{3}} & 1 \\
z_{1} \zeta_{1} & z_{1} & \zeta_{1} & 1 \\
z_{2} \zeta_{2} & z_{2} & \zeta_{2} & 1 \\
z_{3} \zeta_{3} & z_{3} & \zeta_{3} & 1
\end{array}\right]= \\
\frac{1}{2} \operatorname{det}\left[\begin{array}{ccc}
z_{1} & \zeta_{1} & \xi_{2}-\xi_{3} \\
z_{2} & \zeta_{2} & \xi_{2} \\
z_{3} & \zeta_{3} & -\xi_{3}
\end{array}\right] .
\end{gathered}
$$

It is somewhat surprising that the two left-hand-side determinants in the identities above are equal.

\section{The Johnson-Tzitzeica Circles}

Johnson-Tzitzeica. Three circles of equal radii $r$ intersect at a point I, and let $A_{1}, A_{2}$, and $A_{3}$ be the other three points where they intersect pairwise. Show that the circumcircle of triangle $A_{1} A_{2} A_{3}$ has the same radius $r[2,4]$ (Figure 3).

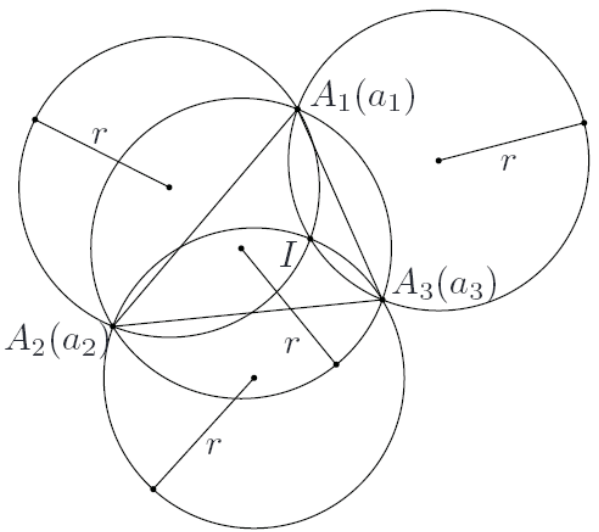

Figure 3: The Johnson-Tzitzeica circles.

With the coordinate system centered in $I$, let $A_{i}$ have coordinate $a_{i}, i=$ $1,2,3$. Any one of the three circles appears as circumcircle to the triangle with vertices two of the points $A_{i}$, and $I$. By (3), the circumcircle of triangle 
$A_{1} A_{2} I$ has radius $\rho_{3}$ such that

$$
\rho_{3}^{2}=\frac{\operatorname{det}\left[\begin{array}{ccc}
a_{1}^{2} & a_{1} & 1 \\
a_{2}^{2} & a_{2} & 1 \\
0 & 0 & 1
\end{array}\right] \operatorname{det}\left[\begin{array}{ccc}
\bar{a}_{1}^{2} & \bar{a}_{1} & 1 \\
\bar{a}_{2}^{2} & \bar{a}_{2} & 1 \\
0 & 0 & 1
\end{array}\right]}{-\left(\operatorname{det}\left[\begin{array}{ccc}
a_{1} & \bar{a}_{1} & 1 \\
a_{2} & \bar{a}_{2} & 1 \\
0 & 0 & 1
\end{array}\right]\right)^{2}}=-\frac{a_{1} a_{2}\left(a_{1}-a_{2}\right) \bar{a}_{1} \bar{a}_{2}\left(\bar{a}_{1}-\bar{a}_{2}\right)}{\left(a_{1} \bar{a}_{2}-a_{2} \bar{a}_{1}\right)^{2}}
$$

with similar expressions for $\rho_{1}^{2}$ and $\rho_{2}^{2}$.

Also, the circumcircle of triangle $A_{1} A_{2} A_{3}$ has radius $\rho$, such that

$$
\rho^{2}=\frac{\operatorname{det}\left[\begin{array}{lll}
a_{1}^{2} & a_{1} & 1 \\
a_{2}^{2} & a_{2} & 1 \\
a_{3}^{2} & a_{3} & 1
\end{array}\right] \operatorname{det}\left[\begin{array}{ccc}
\bar{a}_{1}^{2} & \bar{a}_{1} & 1 \\
\bar{a}_{2}^{2} & \bar{a}_{2} & 1 \\
\bar{a}_{3}^{2} & \bar{a}_{3} & 1
\end{array}\right]}{-\left(\operatorname{det}\left[\begin{array}{lll}
a_{1} & \bar{a}_{1} & 1 \\
a_{2} & \bar{a}_{2} & 1 \\
a_{3} & \bar{a}_{3} & 1
\end{array}\right]\right)}
$$

Making the usual replacements $z_{i}$ and $\zeta_{i}$ for $a_{i}$ and $\bar{a}_{i}, i=1,2,3$, denote now by $\xi_{i}, \eta_{i}, \theta_{i}, \xi, \eta$, and $\theta$ the following determinants:

$$
\begin{gathered}
\xi_{3}:=\operatorname{det}\left[\begin{array}{ccc}
z_{1}^{2} & z_{1} & 1 \\
z_{2}^{2} & z_{2} & 1 \\
0 & 0 & 1
\end{array}\right], \eta_{3}:=\operatorname{det}\left[\begin{array}{ccc}
\zeta_{1}^{2} & \zeta_{1} & 1 \\
\zeta_{2}^{2} & \zeta_{2} & 1 \\
0 & 0 & 1
\end{array}\right], \theta_{3}:=\operatorname{det}\left[\begin{array}{ccc}
z_{1} & \zeta_{1} & 1 \\
z_{2} & \zeta_{2} & 1 \\
0 & 0 & 1
\end{array}\right], \text { etc. } \\
\text { and } \xi:=\operatorname{det}\left[\begin{array}{cccc}
z_{1}^{2} & z_{1} & 1 \\
z_{2}^{2} & z_{2} & 1 \\
z_{3}^{2} & z_{3} & 1
\end{array}\right], \eta:=\operatorname{det}\left[\begin{array}{ccc}
\zeta_{1}^{2} & \zeta_{1} & 1 \\
\zeta_{2}^{2} & \zeta_{2} & 1 \\
\zeta_{3}^{2} & \zeta_{3} & 1
\end{array}\right], \theta:=\operatorname{det}\left[\begin{array}{ccc}
z_{1} & \zeta_{1} & 1 \\
z_{2} & \zeta_{2} & 1 \\
z_{3} & \zeta_{3} & 1
\end{array}\right]
\end{gathered}
$$

Trivially,

$$
\xi=\xi_{1}+\xi_{2}+\xi_{3}, \quad \eta=\eta_{1}+\eta_{2}+\eta_{3}, \quad \text { and } \quad \theta=\theta_{1}+\theta_{2}+\theta_{3} .
$$

From (8), the Johnson-Tzitzeica statement follows as a specialization of either one of the determinant identities

$$
\frac{\theta_{1}^{2}}{\xi_{1}}+\frac{\theta_{2}^{2}}{\xi_{2}}+\frac{\theta_{3}^{2}}{\xi_{3}}=\frac{\theta^{2}}{\xi} \quad \text { or } \quad \frac{\theta_{1}^{2}}{\eta_{1}}+\frac{\theta_{2}^{2}}{\eta_{2}}+\frac{\theta_{3}^{2}}{\eta_{3}}=\frac{\theta^{2}}{\eta},
$$

in the presence of the hypothesis of equal radii, $\rho_{1}=\rho_{2}=\rho_{3}=r$. In (9) obvious restrictions should be imposed on the complex variables $z_{i}, \zeta_{i}$, $i=1,2,3$, such that the denominators involved do not vanish.

\section{An Extension of an IMO Problem}


IMO 2011. Let $A_{1} A_{2} A_{3}$ be a triangle with center of gravity $G$ and with circumcircle $\Gamma$ of circumcenter $O$ and circumradius $r$. Let $l$ be a line tangent to $\Gamma$ at a point $T$, and let $d$ be the line parallel to $l$ and at distance $r / 3$ from $O$. Finally, let $l_{1}, l_{2}$, and $l_{3}$ be the lines obtained by reflecting $l$ in the lines $A_{2} A_{3}, A_{3} A_{1}$, and $A_{1} A_{2}$, respectively (Figure 4).

a) If the triangle $A_{1} A_{2} A_{3}$ is acute, then the lines $l_{1}, l_{2}$, and $l_{3}$ determine $a$ triangle whose circumcircle $\Gamma_{l}$ is such that $\Gamma$ is tangent interior to $\Gamma_{l}$.

b) If the triangle $A_{1} A_{2} A_{3}$ is right with, say, right angle at vertex $A_{1}$, then $l_{2}$ and $l_{3}$ are parallel, and $l_{1}$ is tangent to $\Gamma$ at the point symmetric to $T$ with respect to $A_{2} A_{3}$.

c) If the triangle $A_{1} A_{2} A_{3}$ is obtuse then $\Gamma$ and $\Gamma_{l}$ are tangent exterior if $O$ and $G$ are on the same side of $d$, and $\Gamma_{l}$ is tangent interior to $\Gamma$, if $O$ and $G$ are on opposite sides of $d$ (Figure 4 ). When $G$ is on $d$ then $\Gamma_{l}$ degenerates to a point on $\Gamma$.

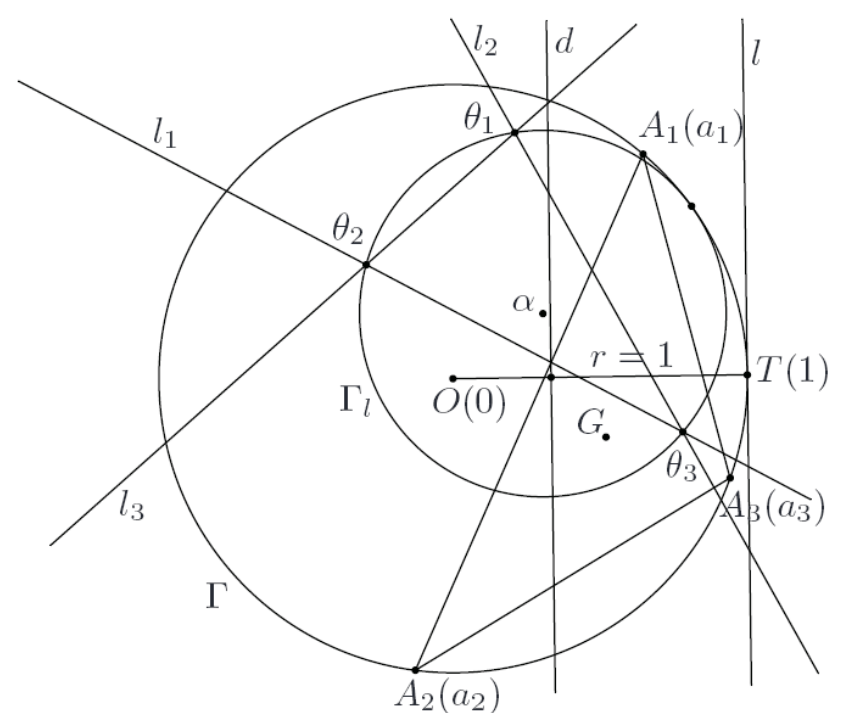

Figure 4: The IMO problem, case $c$ ), interior tangency.

The case $a$ ) was presented at the International Mathematical Olympiad, Amsterdam 2011, as Problem 6. The main difficulty in solving it synthetically consists in understanding the geometry of the two-circle point of tangency. This difficulty disappears when seeking an analytic solution, given the simple characterization of tangent circles in terms of their radii and the distance between their centers.

We can choose a coordinate system such that $O$ has coordinate 0 , distances 
DETERMINANT IDENTITIES AND

are scaled so $r=1$, and $T$ has coordinate 1 . Then $\Gamma$ has equation $z \bar{z}=1, l$ has equation $z+\bar{z}=2$, and $A_{i}, i=1,2,3$, will have coordinates some distinct complex numbers $a_{i}$ of modulus 1 , respectively.

In all the calculations that follow we will make crucial use of the fact that $\bar{a}_{i}=\frac{1}{a_{i}}, i=1,2,3$. Using the formula (5) we see that $l_{3}$ has equation

$$
z+a_{1}^{2} a_{2}^{2} \bar{z}-\left(a_{1}+a_{2}\right)\left(1+a_{1} a_{2}\right)+2 a_{1} a_{2}=0,
$$

and similar equations hold for $l_{1}$ and $l_{2}$.

Consequently, say, the lines $l_{2}$ and $l_{3}$ intersect if and only if $a_{2}+a_{3} \neq 0$. If $a_{2}+a_{3}=0$ then $l_{2}$ and $l_{3}$ are parallel lines, and $A_{2}$ and $A_{3}$ are diametrically opposite on $\Gamma$, i.e., the triangle $A_{1} A_{2} A_{3}$ is right, with the right angle at vertex $A_{1}$. Then $l_{1}$ has equation $z+a_{2}^{4} \bar{z}-2 a_{2}^{2}=0$, which is tangent to $\Gamma$, at the point with coordinate $a_{2}^{2}$. A moment thought shows that this point is the reflection of $T$ in $A_{2} A_{3}$. This proves $b$ ).

Assume further that any two of the three lines $l_{1}, l_{2}$, and $l_{3}$ intersect, i.e., $a_{1}+a_{2} \neq 0, a_{2}+a_{3} \neq 0$, and $a_{3}+a_{1} \neq 0$. Then the lines $l_{2}$ and $l_{3}$ intersect at a point whose coordinate is

$$
\theta_{1}:=\frac{\left(a_{1}-2\right) a_{1} a_{2} a_{3}+\left(a_{1} a_{2}+a_{2} a_{3}+a_{3} a_{1}\right)}{a_{2}+a_{3}} .
$$

There are similar expressions for the coordinates of the other two intersection points, $\theta_{2}$, and $\theta_{3}$. By equation (1) we see that

$$
\begin{aligned}
& \operatorname{det}\left[\begin{array}{lll}
\theta_{1} & \bar{\theta}_{1} & 1 \\
\theta_{2} & \bar{\theta}_{2} & 1 \\
\theta_{3} & \bar{\theta}_{3} & 1
\end{array}\right]= \\
& \frac{\left(a_{1}-a_{2}\right)\left(a_{2}-a_{3}\right)\left(a_{3}-a_{1}\right)}{\left(a_{1}+a_{2}\right)\left(a_{2}+a_{3}\right)\left(a_{3}+a_{1}\right)}\left(a_{1}+a_{2}+a_{3}+\frac{1}{a_{1}}+\frac{1}{a_{2}}+\frac{1}{a_{3}}-2\right),
\end{aligned}
$$

and so the points $\theta_{1}, \theta_{2}$, and $\theta_{3}$ are non-collinear, and define indeed the circle $c\left(\theta_{1}, \theta_{2}, \theta_{3}\right)=\Gamma_{l}$, if and only if $a_{1}+a_{2}+a_{3}+\frac{1}{a_{1}}+\frac{1}{a_{2}}+\frac{1}{a_{3}}-2 \neq 0$, which is equivalent with the center of gravity $G$ of the triangle $A_{1} A_{2} A_{3}$ not falling on $d$. If $G$ belongs to $d$ the circle degenerates to a point, since $\theta_{1}=\theta_{2}=\theta_{3}=$ $-a_{1} a_{2} a_{3}$. By (3), the circle $\Gamma_{l}$ has then center $\alpha$ and radius $\rho$ such that

$$
-\frac{\operatorname{det}\left[\begin{array}{lll}
\theta_{1} \bar{\theta}_{1} & \theta_{1} & 1 \\
\theta_{2} \bar{\theta}_{2} & \theta_{2} & 1 \\
\theta_{3} \bar{\theta}_{3} & \theta_{3} & 1
\end{array}\right]}{\operatorname{det}\left[\begin{array}{lll}
\theta_{1} & \bar{\theta}_{1} & 1 \\
\theta_{2} & \bar{\theta}_{2} & 1 \\
\theta_{3} & \bar{\theta}_{3} & 1
\end{array}\right]}=\frac{\left(a_{1} a_{2}+a_{2} a_{3}+a_{3} a_{1}-a_{1} a_{2} a_{3}\right)^{2}}{\left(a_{1}+a_{2}\right)\left(a_{2}+a_{3}\right)\left(a_{3}+a_{1}\right)},
$$




$$
\text { and } \quad \begin{aligned}
\rho^{2}= & \frac{\operatorname{det}\left[\begin{array}{lll}
\theta_{1}^{2} & \theta_{1} & 1 \\
\theta_{2}^{2} & \theta_{2} & 1 \\
\theta_{3}^{2} & \theta_{3} & 1
\end{array}\right] \operatorname{det}\left[\begin{array}{ccc}
\bar{\theta}_{1}^{2} & \bar{\theta}_{1} & 1 \\
\bar{\theta}_{2}^{2} & \bar{\theta}_{2} & 1 \\
\bar{\theta}_{3}^{2} & \bar{\theta}_{3} & 1
\end{array}\right]}{-\left(\operatorname{det}\left[\begin{array}{ccc}
\theta_{1} & \bar{\theta}_{1} & 1 \\
\theta_{2} & \bar{\theta}_{2} & 1 \\
\theta_{3} & \bar{\theta}_{3} & 1
\end{array}\right]\right)^{2}} \\
= & \left(\frac{\left(a_{1}+a_{2}+a_{3}-2\right) a_{1} a_{2} a_{3}+\left(a_{1} a_{2}+a_{2} a_{3}+a_{3} a_{1}\right)}{\left(a_{1}+a_{2}\right)\left(a_{2}+a_{3}\right)\left(a_{3}+a_{1}\right)}\right)^{2} .
\end{aligned}
$$

Noticing now that the quantities

$\sigma:=a_{1}+a_{2}+a_{3}+\frac{1}{a_{1}}+\frac{1}{a_{2}}+\frac{1}{a_{3}}-2$ and $\pi:=\left(1+\frac{a_{1}}{a_{2}}\right)\left(1+\frac{a_{2}}{a_{3}}\right)\left(1+\frac{a_{3}}{a_{1}}\right)$

are real, $\pi \neq 0$, while the quantity

$$
\epsilon:=\left(a_{1} a_{2}+a_{2} a_{3}+a_{3} a_{1}-a_{1} a_{2} a_{3}\right) \overline{\left(a_{1} a_{2}+a_{2} a_{3}+a_{3} a_{1}-a_{1} a_{2} a_{3}\right)}
$$

is real, non-negative, from (12) and (13) we conclude that

$$
\alpha \bar{\alpha}=\left(\frac{\epsilon}{\pi}\right)^{2}, \quad \rho^{2}=\left(\frac{\sigma}{\pi}\right)^{2}, \quad \text { and most importantly } \frac{\epsilon}{\pi}+\frac{\sigma}{\pi}=1 .
$$

The first two equations (14) show that the circle $\Gamma_{l}$ has radius $\rho=\frac{|\sigma|}{|\pi|}$, while the distance between the centers of the circles $\Gamma$ and $\Gamma_{l}$ is $|\alpha|=\frac{\epsilon}{|\pi|}$.

The last of the equations (14) holds the key to the solution of our problem in cases $a$ ) and $c$ ) as soon as the geometric meaning of the signs of the the real quantities $\sigma$ and $\pi$ is decoded.

Looking at its expression, it is clear that $\sigma$ is positive when $O$ and $G$ are on opposite sides of $d$, negative when they are on the same side, and vanishes when $G$ belongs to $d$.

The interpretation of the sign of $\pi$ is subtler: First of all, the triangle $A_{1} A_{2} A_{3}$ has an acute, respectively obtuse, angle at, say, vertex $A_{1}$ if and only if $\omega_{1}:=\left(a_{2}-a_{1}\right)\left(\bar{a}_{3}-\bar{a}_{1}\right)+\left(a_{3}-a_{1}\right)\left(\bar{a}_{2}-\bar{a}_{1}\right)>0$, respectively $<0$. If follows that the triangle itself is acute or obtuse depending on the sign of the product $\omega_{1} \omega_{2} \omega_{3}$. An easy calculation shows that

$$
\omega_{1} \omega_{2} \omega_{3}=-\pi \omega \bar{\omega}
$$

where

$$
\omega:=i\left(1-\frac{a_{1}}{a_{2}}\right)\left(1-\frac{a_{2}}{a_{3}}\right)\left(1-\frac{a_{3}}{a_{1}}\right) \neq 0 .
$$


DETERMINANT IDENTITIES AND

As a result, triangle $A_{1} A_{2} A_{3}$ is acute if $\pi<0$ and obtuse if $\pi>0$.

Now, the conclusion in cases $a$ ) and $c$ ) follows easily from the facts above, since two circles are tangent interior/exterior if and only if the distance between their centers equals the difference/sum of their radii.

\section{Conclusions}

The plane geometry of lines and circles translates neatly into determinant identities, via complex analytic geometry. The readers are encouraged to run their favorite geometry problems through this machinery.

In the process an obvious question will emerge: How to find the appropriate determinant identities? Clearly, the main clues are contained in the actual statement of a given problem. For the most direct approach, if many defining elements in the problem are points on a given circle then the coordinate system should be centered at the center of the circle, and the unit distance should be taken to be its radius, like in the IMO problem. This choice introduces the least number of variables. Indeed, since lines and circles as defined by equations (1) and (2) contain complex variables and their conjugates, when a quantity belongs to the unit circle, its conjugate, being equal to its reciprocal, does not require a new variable. Also, it is important not to make coordinate choices which interfere with the symmetry and cyclicity of par quantities.

Beyond that, the sought after identities must relate the 'hypothesis determinants' to the 'conclusion determinants'. Finding them is pretty much a trial and error exercise, greatly helped by reader intuition and creativity. Usually, the 'correct' identities afford elegant verifications, via determinant properties. However, it pays to know that a computer algebra system capable of carrying out symbolic calculations, like Maple or Mathematica, can help test, verify, or even discover, potential identities.

\section{References}

[1] J. Conway, Functions of One Complex Variable, 2nd Edition, Springer, New York, (1978).

[2] R. Johnson, A Circle Theorem, Amer. Math. Monthly, 23, No. 5, 161$162,(1916)$.

[3] R. Todor, A Computational Solution of Question 6 from IMO 2011, Gaz. Mat. B, CXVII, No. 1, 1-7, (2012).

[4] G. Tzitzeica, Problems in Geometry, 6th Edition (Romanian), Editura Tehnică, Bucharest, (1961). 
DETERMINANT IDENTITIES AND

Nicolae ANGHEL

Department of Mathematics,

University of North Texas,

Denton TX, 76203

Email: anghel@unt.edu 
DETERMINANT IDENTITIES AND

THE GEOMETRY OF LINES AND CIRCLES 